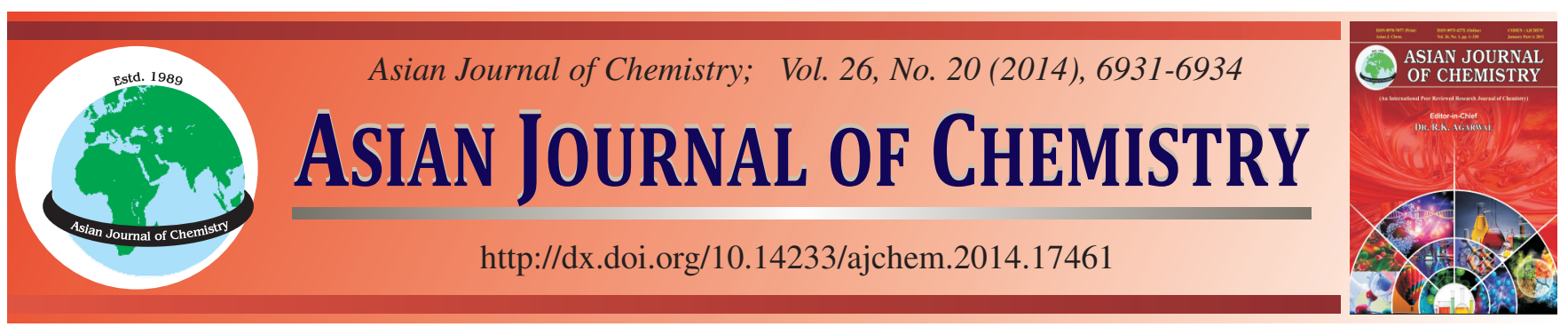

\title{
Synthesis and Characterization of Diazenyl-1,3-diphenylpropane-1,3-dione and 5-[Hydroxy(phenyl)methyl]-4-phenylpyrimidine Derivatives
}

\author{
ZÜlbiYe ÖNal ${ }^{*}$, Ayhan ÖZalp, Ilhan ÖZer Ilhan and GüZIN Aslan
}

Department of Chemistry, Faculty of Sciences, Erciyes University, 38039 Kayseri, Turkey

*Corresponding author: Fax: +90 3524374931 E-mail: zulbiye@erciyes.edu.tr

\section{INTRODUCTION}

Pyrimidine derivatives are well known pharmacophores exhibiting a wide spectrum of pharmacological activities. They show various interesting pharmacological properties including antiviral, antibacterial, antitumour and antiflammatory effects. Some of them are frequently encountered in many drugs used for the treatment of hypothyroidy, hypertension, cancer chemotherapy and HIV infection ${ }^{1-5}$. There are published reports about the synthesis of some pyrimidine derivatives from furan-2,3diones $^{6-11}$ (Scheme-I). Also conformational analysis and quantum chemical calculations were carried out by means of MMP2, CNDO, MNDO and AM1 approximation methods for a series of compounds which are functionalized pyrimidine derivatives ${ }^{8,9}$. The $\mathrm{N}$-aminopyrimidine derivatives 1a-f which exhibit a free $\mathrm{N}-\mathrm{NH}_{2}$ moiety were applied in several subsequent reactions. Recently, the reactions of $\mathrm{N}$-aminopyrimidine<smiles>[Y]C(N)=N/N=C(\C)c1ccccc1</smiles>

Scheme-I: Synthesis of $N$-aminopyrimidine derivatives 1a-f derivatives 1a-f with several anhydrides, isocyanates, isothiocyanates and 1,3-dicarbonyl compounds have been reported in different solvents at various temperatures ${ }^{12-17}$.

In view of these important properties, we decided both to prove the producibility of the reactions of $N$-aminopyrimidine derivatives 1a-f with $\beta$-diketones and sodium borohydride and to extend our investigations related to the preparation of new heterocycles which include the pyrimidine ring in their structures. In the present study, the synthesis of some new diazenyl1,3-diphenylpropane-1,3-dione derivatives 2a-f were carried out (Scheme-II). In addition, in this work, a series of novel 5[hydroxy(4-methylphenyl)methyl]-4-(methylphenyl)pyrimidine derivatives 3a-f were synthesized by treating $\mathrm{N}$-aminopyrimidine derivatives 1a-f with $\mathrm{NaBH}_{4}$ (Scheme-III). The newly synthesized compounds were established on the basis of elemental analysis, IR, ${ }^{1} \mathrm{H}$ and ${ }^{13} \mathrm{C}$-NMR spectral studies.

\section{EXPERIMENTAL}

Melting points were determined on an Electrothermal 9200 apparatus and are uncorrected. Microanalyses were performed on a Leco-932 CHNS-O Elemental Analyzer, model 1108. A Shimadzu FT-IR-8400 model spectrophotometer was used for IR spectra (4000-400 $\mathrm{cm}^{-1}$ region), using ATR techniques. The ${ }^{1} \mathrm{H}$ - and ${ }^{13} \mathrm{C}-\mathrm{NMR}$ spectra were measured with a Bruker Avance III $400 \mathrm{MHz}$ spectrometer and the chemical shifts were recorded in ppm units. After completion of the reactions, the solvents were evaporated with a rotary evaporator (Buchi RE model 111). The reactions were followed by TLC using a DC Alufolien Kieselgel 60 F254 Merck and a Camag 
TLC lamp (254/366 nm). Solvents and all other chemical reagents were purchased from commercial suppliers and were of reagent grade quality. Solvents were dried by refluxing with the appropriate drying agents and distilled before use.

General procedure for the synthesis of diazenyl-1,3diphenylpropane-1,3-dione derivatives (2a-f): 1-Amino-5benzoyl-4-phenyl-1 $\mathrm{H}$-pyrimidine-2-ones/-thiones (1a-f) (3.43 $\mathrm{mmol}$ ) in $30 \mathrm{~mL}$ of EtOH was warmed until dissolved. The mixture was cooled to room temperature, $0.9 \mathrm{~mL}(10.29 \mathrm{mmol})$ of concentrated $\mathrm{HCl}$ was added and the reaction mixture was cooled to a temperature below $5^{\circ} \mathrm{C}$. And then the mixture was added to an ice cold solution of $\mathrm{NaNO}_{2}(3.43 \mathrm{mmol})$ in water $(10 \mathrm{~mL})$ drop wise with constant stirring. Temperature was maintained below $5{ }^{\circ} \mathrm{C}$. The diazonium salts of (1a-f) were reacted with dibenzoylmethane (molar ratio1:1) in the presence of $\mathrm{NaOH}$. The reaction mixture continuously stirring for $5-9 \mathrm{~h}$ at $60-70{ }^{\circ} \mathrm{C}$. Then the reaction mass was poured into ice-cold water and acidified. The solid obtained (2a-f) was filtered and recrystallized from alcohols and dried on $\mathrm{P}_{2} \mathrm{O}_{5}$.

2-[(E)-(5-Benzoyl-2-oxo-4-phenylpyrimidine-1 $(2 \mathrm{H})$ yl)diazenyl]-1,3-diphenylpropane-1,3-dione (2a): The obtained product $\mathbf{2 a}$ was crystallized in $n$-butanol and dried on $\mathrm{P}_{2} \mathrm{O}_{5}$ and was obtained in $50 \%$ yield as a colourless mass; m.p. $248-249^{\circ} \mathrm{C}$. IR: $\left(\mathrm{KBr}, v_{\max }, \mathrm{cm}^{-1}\right)$ : 3050 (aromatic C-H), 2930 (aliphatic C-H), 1730, 1680, $1650(\mathrm{C}=\mathrm{O}), 1620(\mathrm{C}=\mathrm{C}$ and $\mathrm{C}=\mathrm{N}$ ), 800-640 (pyrimidine ring); ${ }^{1} \mathrm{H}$ NMR $(400 \mathrm{MHz}$, DMSO- $\left.d_{6}\right): \delta=14.90(\mathrm{~s}, 1 \mathrm{H}$, enol OH), 7.36-7.02 (m, 20H, Ar-H), 6.08 (s, 1H, C-H); ${ }^{13} \mathrm{C}-\mathrm{NMR}\left(400 \mathrm{MHz},\left(\mathrm{DMSO}-d_{6}\right)\right.$ : $\delta=190.80-188.10$ (benzoyl groups, $\mathrm{C}=\mathrm{O}$ ), 153.15 (pyrimidine ring, $\mathrm{C}=\mathrm{O}$ ), 150.05-110.42 (aromatic $\mathrm{C}$ ), $74.82 \mathrm{ppm}(\mathrm{C}-\mathrm{H})$. Anal. Calcd. for $\mathrm{C}_{32} \mathrm{H}_{22} \mathrm{~N}_{4} \mathrm{O}_{4}$ : C, 72.99; H, 4.21; N, 10.64. Found: C, 72.89; H, 4.15; N, 10.50 .

2-[(E)-(5-Benzoyl-2-thioxo-4-phenylpyrimidine-1(2H)yl)diazenyl]-1,3-diphenylpropane-1,3-dione (2b): The obtained product $\mathbf{2} \mathbf{b}$ was crystallized in $n$-butanol and dried on $\mathrm{P}_{2} \mathrm{O}_{5}$ and was obtained in $50 \%$ yield as a yellow mass; m.p. $255-256{ }^{\circ} \mathrm{C}$. IR (KBr, $\left.v_{\max }, \mathrm{cm}^{-1}\right)$ : 3050 (aromatic $\mathrm{C}-\mathrm{H}$ ), 2930 (aliphatic $\mathrm{C}-\mathrm{H}), 1735$ and $1645(\mathrm{C}=\mathrm{O}), 1620(\mathrm{C}=\mathrm{C}$ and $\mathrm{C}=\mathrm{N}), 1245(\mathrm{C}=\mathrm{S}), 800-620 \mathrm{~cm}^{-1}$ (pyrimidine ring ); ${ }^{1} \mathrm{H}$ NMR $\left(400 \mathrm{MHz}, \mathrm{DMSO}-d_{6}\right): \delta=14.80(\mathrm{~s}, 1 \mathrm{H}$, enol OH$), 7.48-7.02$ (m, 20H, Ar-H), 5.98 (s, 1H, C-H); ${ }^{13} \mathrm{C}$ NMR (400 MHz, (DMSO- $d_{6}$ ): $\delta=191.50-187.10$ (benzoyl groups, $\mathrm{C}=\mathrm{O}$ ), 170.15 (pyrimidine ring, $\mathrm{C}=\mathrm{S}$ ), 151.05-110.00 (aromatic C), 74.52 ppm (C-H). Anal. Calcd. for $\mathrm{C}_{32} \mathrm{H}_{22} \mathrm{~N}_{4} \mathrm{O}_{3} \mathrm{~S}$ : C, 70.83; H, 4.09; N, 10.33; S, 5.91. Found: C, 70.71; H, 4.01; N, 10.25; S, 5.80.

2-\{(E)-[5-(4-Methylbenzoyl)-4-(4-methylphenyl)-2oxopyrimidine-1(2H)-yl]diazenyl\}-1,3-diphenyl- propane1,3-dione (2c): The obtained product $2 \mathrm{c}$ was crystallized in ethanol and dried on $\mathrm{P}_{2} \mathrm{O}_{5}$ and was obtained in $57 \%$ yield as a colourless mass; m.p. $270-271^{\circ} \mathrm{C}$. IR ( $\left.\mathrm{KBr}, \mathrm{v}_{\max }, \mathrm{cm}^{-1}\right)$ : 3040 (aromatic C-H), 2930 (aliphatic C-H), 1735, 1685 and 1655 $(\mathrm{C}=\mathrm{O}), 1620(\mathrm{C}=\mathrm{C}$ and $\mathrm{C}=\mathrm{N}), 1245(\mathrm{C}=\mathrm{S}), 800-640$ (pyrimidine ring ); ${ }^{1} \mathrm{H}$ NMR (400 MHz, DMSO- $\left.d_{6}\right): \delta=14.50(\mathrm{~s}, 1 \mathrm{H}$, enol OH), 7.56-7.05 (m, 18H, Ar-H), 6.08 (s, 1H, C-H); 2.14, $2.05\left(\mathrm{~s}, 6 \mathrm{H}, 2 \times \mathrm{CH}_{3}\right) ;{ }^{13} \mathrm{C} \mathrm{NMR}\left(400 \mathrm{MHz},\left(\mathrm{DMSO}-d_{6}\right): \delta=\right.$ 190.80-188.10 ( $\mathrm{C}=\mathrm{O}$, benzoyl groups), $153.15(\mathrm{C}=\mathrm{O}$, pyrimidine ring), 150.05-110.42 (aromatic C), $74.82(\mathrm{C}-\mathrm{H}), 22.15$,

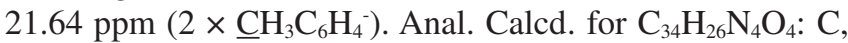
73.63; H, 4.73; N, 10.10. Found: C, 73.45; H, 4.54; N, 10.22.
2-\{(E)-[5-(4-Methylbenzoyl)-4-(4-methylphenyl)-2thioxopyrimidine-1(2H)-yl]diazenyl $\}$-1,3-diphenylpropane-1,3-dione (2d): The obtained product 2d was crystallized in ethanol and dried on $\mathrm{P}_{2} \mathrm{O}_{5}$ and was obtained in $58 \%$ yield as a yellow mass; m.p. $285-286{ }^{\circ} \mathrm{C}$. IR $\left(\mathrm{KBr}, \mathrm{v}_{\max }\right.$, $\mathrm{cm}^{-1}$ ): 3040 (aromatic C-H), 2920 (aliphatic C-H), 1735, 1645 $(\mathrm{C}=\mathrm{O}), 1620(\mathrm{C}=\mathrm{C}$ and $\mathrm{C}=\mathrm{N}), 1245(\mathrm{C}=\mathrm{S}), 800-640$ (pyrimidine ring); ${ }^{1} \mathrm{H}$ NMR (400 MHz, DMSO- $\left.d_{6}\right): \delta=14.62(\mathrm{~s}, 1 \mathrm{H}$, enol OH), 7.70-7.05 (m, 18H, Ar-H), 6.01 (s, 1H, C-H); 2.15, $2.05\left(\mathrm{~s}, 6 \mathrm{H}, 2 \times \mathrm{CH}_{3}\right) ;{ }^{13} \mathrm{C} \mathrm{NMR}\left(400 \mathrm{MHz},\left(\mathrm{DMSO}-d_{6}\right): \delta=\right.$ 190.80-188.10 (C=O, benzoyl groups), 170.15 (C=S, pyrimidine ring), 158.15 (pyrimidine ring, $\mathrm{C}=\mathrm{S}$ ), 150.05-110.42 (aromatic C), $73.82(\mathrm{C}-\mathrm{H}), 22.15,21.64 \mathrm{ppm}\left(2 \times \mathrm{CH}_{3} \mathrm{C}_{6} \mathrm{H}_{4}{ }^{-}\right)$. Anal. Calcd. for $\mathrm{C}_{34} \mathrm{H}_{26} \mathrm{~N}_{4} \mathrm{O}_{3} \mathrm{~S}$ : C, 71.56; H, 4.59; N, 9.82; S, 7.63. Found: C, 71.38; H, 4.42; N, 9.64; S, 7.50.

2-\{(E)-[5-(4-Methoxybenzoyl)-4-(4-methoxyphenyl)-2oxopyrimidine-1(2H)-yl]diazenyl\}-1,3-diphenylpropane1,3-dione (2e): The obtained product $2 \mathrm{e}$ was crystallized in ethanol and dried on $\mathrm{P}_{2} \mathrm{O}_{5}$ and was obtained in $63 \%$ yield as a colourless mass; m.p. $273-274{ }^{\circ} \mathrm{C}$. IR $\left(\mathrm{KBr}, \mathrm{v}_{\max }, \mathrm{cm}^{-1}\right)$ : 3040 (aromatic C-H ), 2935 (aliphatic C-H), 1730, $1650 \mathrm{C}=\mathrm{O}$ ), 1620 $\left(\mathrm{C}=\mathrm{C}\right.$ and $\mathrm{C}=\mathrm{N}$ ), 800-640 (pyrimidine ring); ${ }^{1} \mathrm{H}$ NMR (400 MHz, DMSO- $\left.d_{6}\right): \delta=14.56(\mathrm{~s}, 1 \mathrm{H}$, enol OH), 7.78-7.05 (m, 18H, Ar-H), 6.01 (s, 1H, C-H); 3.89, 3.86 (s, 6H, $\left.2 \times \mathrm{CH}_{3}\right)$; ${ }^{13} \mathrm{C}$ NMR (400 MHz, (DMSO- $\left.d_{6}\right): \delta=190.80-189.10(\mathrm{C}=\mathrm{O}$, benzoyl groups), 152.15 ( $\mathrm{C}=\mathrm{O}$, pyrimidine ring), 151.05115.42 (aromatic C), $73.82(\mathrm{C}-\mathrm{H}), 55.90,54.89 \mathrm{ppm}(2 \times$ $\left.\mathrm{CH}_{3} \mathrm{OC}_{6} \mathrm{H}_{4}-\right)$. Anal. Calcd. for $\mathrm{C}_{34} \mathrm{H}_{26} \mathrm{~N}_{4} \mathrm{O}_{6}$ : C, 69.62; $\mathrm{H}, 4.47$; N, 9.55. Found: C, 69.55; H, 4.36; N, 9.42.

2-\{(E)-[5-(4-methoxybenzoyl)-4-(4-methoxyphenyl)-2thioxopyrimidine-1(2H)-yl]diazenyl \}-1,3-diphenylpropane-1,3-dione (2f): The obtained product $2 \mathbf{f}$ was crystallized in $n$-butanol and dried on $\mathrm{P}_{2} \mathrm{O}_{5}$ and was obtained in 67 $\%$ yield as a light yellow mass; m.p. $288-289{ }^{\circ} \mathrm{C}$. IR $(\mathrm{KBr}$, $\left.V_{\max }, \mathrm{cm}^{-1}\right): 3040$ (aromatic C-H), 2935 (aliphatic C-H), 1730$1650 \mathrm{C}=\mathrm{O}), 1620(\mathrm{C}=\mathrm{C}$ and $\mathrm{C}=\mathrm{N}), 800-640$ (pyrimidine ring); ${ }^{1} \mathrm{H}$ NMR $\left(400 \mathrm{MHz}, \mathrm{DMSO}-d_{6}\right): \delta=14.52(\mathrm{~s}, 1 \mathrm{H}$, enol OH$)$, 7.87-7.05 (m, 18H, Ar-H), 6.03 (s, 1H, C-H); 3.88, 3.84 (s, $\left.6 \mathrm{H}, 2 \times \mathrm{CH}_{3}\right) ;{ }^{13} \mathrm{C} \mathrm{NMR}\left(400 \mathrm{MHz},\left(\mathrm{DMSO}-d_{6}\right): \delta=189.80-\right.$ $188.10(\mathrm{C}=\mathrm{O}$, benzoyl groups $), 170.30(\mathrm{C}=\mathrm{S}$, pyrimidine ring $)$, 155.01-112.22 (aromatic C), $72.82(\mathrm{C}-\mathrm{H}), 55.40,54.90 \mathrm{ppm}$ $\left(2 \times \mathrm{CH}_{3} \mathrm{OC}_{6} \mathrm{H}_{4}-\right)$. Anal. Calcd. for $\mathrm{C}_{34} \mathrm{H}_{26} \mathrm{~N}_{4} \mathrm{O}_{5} \mathrm{~S}: \mathrm{C}, 67.76 ; \mathrm{H}$, $4.35 ;$ N, 9.30; S, 5.32. Found: C, 67.58; H, 4.26; N, 9.15; S, 5.19 .

General procedure for the synthesis of 5-[hydroxy(phenyl)methyl]-4-phenylpyrimidine derivatives (3a-f): To a solution of 1-amino-5-benzoyl-4-phenyl-1H-pyrimidine-2ones/-thiones (1a-f) $(0.4 \mathrm{~g}, 1.36 \mathrm{mmol})$ in EtOH $(50 \mathrm{~mL})$ was added $\mathrm{NaBH}_{4}(0.051 \mathrm{~g}, 1.36 \mathrm{mmol})$ in portions at $0.5 \mathrm{~h}$ intervals by stirring. The reaction mixture was refluxed for $1 \mathrm{~h}$. It was then poured into ice-cold water and $5 \mathrm{~mL}$ of concentrated hydrochloric acid, after which it was extracted with $\mathrm{CHCl}_{3}$. The $\mathrm{CHCl}_{3}$ was evaporated in vacuo and crude product recrystallized from a suitable solvent (i.e. $n$-butanol or ethanol) and dried on $\mathrm{P}_{2} \mathrm{O}_{5}$.

1-Amino-5-[hydroxy(phenyl)methyl]-4-phenylpyrimidine-2(1H)-one (3a): The obtained product 3a was crystallized in $n$-butanol and dried on $\mathrm{P}_{2} \mathrm{O}_{5}$ and was obtained in 54 $\%$ yield as a colourless mass; m.p. $255-256{ }^{\circ} \mathrm{C}$. IR ( $\mathrm{KBr}, \mathrm{v}_{\max }$, 
$\left.\mathrm{cm}^{-1}\right): 3300(\mathrm{O}-\mathrm{H}), 3200(\mathrm{~N}-\mathrm{H}), 3058$ (aromatic C-H), 1650 $(\mathrm{C}=\mathrm{O}), 1620(\mathrm{C}=\mathrm{C}$ and $\mathrm{C}=\mathrm{N}), 800-640$ (pyrimidine ring); ${ }^{1} \mathrm{H}$ NMR (400 MHz, DMSO-d $): \delta=10.51(\mathrm{~s}, 1 \mathrm{H}$, pyrimidine C-H), 7.38-7.02 (m, 10H, Ar-H), 5.91 (s, 2H, N-H); 5.74, (s, $1 \mathrm{H}, \mathrm{C}-\mathrm{H}) ;{ }^{13} \mathrm{C}$ NMR $\left(400 \mathrm{MHz},\left(\mathrm{DMSO}-d_{6}\right): \delta=152.80(\mathrm{C}=\mathrm{O}\right.$, pyrimidine ring), 143.15-117.42 (aromatic C), 73.08 ppm $(\mathrm{CH}-$ $\mathrm{OH})$. Anal. Calcd. for for $\mathrm{C}_{17} \mathrm{H}_{15} \mathrm{~N}_{3} \mathrm{O}_{2}$ : C, 69.61; H, 5.15; N, 14.33. Found: C, 69.49; H, 5.30; N, 14.15.

1-Amino-5-[hydroxy(phenyl)methyl]-4-phenylpyrimidine-2(1H)-thione (3b): The obtained product $\mathbf{3 b}$ was crystallized in EtOH and dried on $\mathrm{P}_{2} \mathrm{O}_{5}$ and was obtained in $59 \%$ yield as a light yellow mass; m.p. $235-236{ }^{\circ} \mathrm{C}$. IR $(\mathrm{KBr}$, $\left.\mathrm{v}_{\max }, \mathrm{cm}^{-1}\right): 3300(\mathrm{O}-\mathrm{H}), 3220(\mathrm{~N}-\mathrm{H}), 3050$ (aromatic $\left.\mathrm{C}-\mathrm{H}\right)$, 1620-1560 $(\mathrm{C}=\mathrm{C}$ and $\mathrm{C}=\mathrm{N}), 1220(\mathrm{C}=\mathrm{S}), 800-640$ (pyrimidine ring); ${ }^{1} \mathrm{H}$ NMR (400 MHz, DMSO- $\left.d_{6}\right): \delta=10.60(\mathrm{~s}, 1 \mathrm{H}$, pyrimidine C-H), 7.38-7.10 (m, 10H, Ar-H), 5.90 (s, 1H, O$\mathrm{H}) ; 5.85$, (s, 2H, N-H); 5.75 (s, 1H, C-H) ${ }^{13} \mathrm{C} \mathrm{NMR}(400 \mathrm{MHz}$, $\left(\mathrm{DMSO}-d_{6}\right): \delta=148.80(\mathrm{C}=\mathrm{S}$, pyrimidine ring), 150.15-110.42 (aromatic C), $73.01 \mathrm{ppm}(\mathrm{CH}-\mathrm{OH})$. Anal. Calcd. for for $\mathrm{C}_{17} \mathrm{H}_{15} \mathrm{~N}_{3} \mathrm{OS}$ : C, 66.00; H, 4.89; N, 13.58; S, 10.36. Found: C, 66.17; H, 4.98; N, 13.40, S, 10.08 .

1-Amino-5-[hydroxy(4-methylphenyl)methyl]-4(methylphenyl)pyrimidine-2(1H)-one $(3 \mathrm{c})$ : The obtained product $3 \mathbf{c}$ was crystallized in $\mathrm{EtOH}$ and dried on $\mathrm{P}_{2} \mathrm{O}_{5}$ and was obtained in $64 \%$ yield as a colourless mass; m.p. 247$248{ }^{\circ} \mathrm{C}$. IR (KBr, $\left.v_{\max }, \mathrm{cm}^{-1}\right): 3300(\mathrm{O}-\mathrm{H}), 3220(\mathrm{~N}-\mathrm{H}), 3050$ (aromatic C-H), 1620-1560 ( $\mathrm{C}=\mathrm{C}$ and $\mathrm{C}=\mathrm{N}), 1220(\mathrm{C}=\mathrm{S}), 800$ 640 (pyrimidine ring); ${ }^{1} \mathrm{H} \mathrm{NMR}\left(400 \mathrm{MHz}, \mathrm{DMSO}-d_{6}\right): \delta=$ 10.55 (s, 1H, pyrimidine C-H), 7.38-7.10 (m, 8H, Ar-H), 5.90 (s, 1H, O-H); 5.85, (s, 2H, N-H); $5.75(\mathrm{~s}, 1 \mathrm{H}, \mathrm{C}-\mathrm{H}) ; 2.27$ (s, $\left.6 \mathrm{H}, 2 \times \mathrm{CH}_{3}\right) ;{ }^{13} \mathrm{C}$ NMR (400 MHz, (DMSO- $\left.d_{6}\right): \delta=151.80$ $(\mathrm{C}=\mathrm{O}$, pyrimidine ring), 152.15-109.42 (aromatic C), 73.04

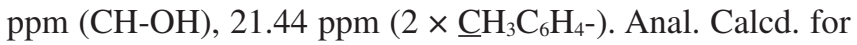
for $\mathrm{C}_{19} \mathrm{H}_{19} \mathrm{~N}_{3} \mathrm{O}_{2}$ : C, 71.01; H, 5.96; N, 13.08. Found: C, 71.20; $\mathrm{H}, 5.80 ; \mathrm{N}, 13.21$.

1-Amino-5-[hydroxy(4-methylphenyl)methyl]-4(methylphenyl)pyrimidine-2(1H)-thione (3d): The obtained product $\mathbf{3 d}$ was crystallized in $\mathrm{EtOH}$ and dried on $\mathrm{P}_{2} \mathrm{O}_{5}$ and was obtained in $69 \%$ yield as a yellow mass; m.p. $222-223{ }^{\circ} \mathrm{C}$. IR $\left(\mathrm{KBr}, v_{\max }, \mathrm{cm}^{-1}\right): 3300(\mathrm{O}-\mathrm{H}), 3221(\mathrm{~N}-\mathrm{H}), 3040$ (aromatic $\mathrm{C}-\mathrm{H}), 1620-1560(\mathrm{C}=\mathrm{C}$ and $\mathrm{C}=\mathrm{N}), 800-640$ (pyrimidine ring); ${ }^{1} \mathrm{H}$ NMR $\left(400 \mathrm{MHz}, \mathrm{DMSO}-d_{6}\right): \delta=10.40(\mathrm{~s}, 1 \mathrm{H}$, pyrimidine C-H), 7.48-7.05 (m, 8H, Ar-H), 5.80 (s, 1H, O-H); 5.85, (s, $2 \mathrm{H}, \mathrm{N}-\mathrm{H}) ; 5.74$ (s, 1H, C-H); $2.27\left(\mathrm{~s}, 6 \mathrm{H}, 2 \times \mathrm{CH}_{3}\right) ;{ }^{13} \mathrm{C} \mathrm{NMR}$ (400 MHz, (DMSO- $\left.d_{6}\right): \delta=170.80(\mathrm{C}=\mathrm{S}$, pyrimidine ring), 152.15-109.42 (aromatic C), 73.12 ppm (CH-OH), 21.44 ppm $\left(2 \times \mathrm{CH}_{3} \mathrm{C}_{6} \mathrm{H}_{4}-\right)$. Anal. Calcd. for for $\mathrm{C}_{19} \mathrm{H}_{19} \mathrm{~N}_{3} \mathrm{OS}: \mathrm{C}, 67.63$; H, 5.68; N, 12.45; S, 9.50. Found: C, 67.50; H, 5.59; N, 12.32; S, 9.62 .

1-Amino-5-[hydroxy(4-methoxyphenyl)methyl]-4(methoxyphenyl)pyrimidine-2(1H)-one (3e): The obtained product $3 \mathbf{e}$ was crystallized in $n$-butanol and dried on $\mathrm{P}_{2} \mathrm{O}_{5}$ and was obtained in $58 \%$ yield as a colourless mass; m.p. 261-262 ${ }^{\circ} \mathrm{C}$. IR (KBr, $\left.v_{\max }, \mathrm{cm}^{-1}\right): 3300(\mathrm{O}-\mathrm{H}), 3221(\mathrm{~N}-\mathrm{H})$, 3040 (aromatic C-H), 2940 (aliphatic C-H), 1620-1560 (C=C and $\mathrm{C}=\mathrm{N}$ ), 800-640 (pyrimidine ring); ${ }^{1} \mathrm{H}$ NMR (400 MHz, DMSO- $\left.d_{6}\right): \delta=10.42(\mathrm{~s}, 1 \mathrm{H}$, pyrimidine $\mathrm{C}-\mathrm{H}), 7.78-6.85(\mathrm{~m}$, $8 \mathrm{H}, \mathrm{Ar}-\mathrm{H}), 5.81$ (s, 1H, O-H); 5.79, (s, 2H, N-H); 5.69 (s, 1H, $\mathrm{C}-\mathrm{H}) ; 3.89,3.87\left(\mathrm{~d}, 6 \mathrm{H}, 2 \times \mathrm{CH}_{3}\right) ;{ }^{13} \mathrm{C} \mathrm{NMR}(400 \mathrm{MHz}$,
$\left(\mathrm{DMSO}-d_{6}\right): \delta=152.80(\mathrm{C}=\mathrm{O}$, pyrimidine ring $), 146.15-$ 110.22 (aromatic C), $75.07(\mathrm{CH}-\mathrm{OH}), 54.64\left(2 \times \mathrm{CH}_{3} \mathrm{OC}_{6} \mathrm{H}_{4} 4^{-}\right)$. Anal. Calcd. for for $\mathrm{C}_{19} \mathrm{H}_{19} \mathrm{~N}_{3} \mathrm{O}_{4}$ : C, 64.58; H, 5.42; N, 11.89 . Found: C, 64.45; H, 5.60; N, 11.70.

1-Amino-5-[hydroxy(4-methoxyphenyl)methyl]-4(methoxyphenyl)pyrimidine-2(1H)-thione (3f): The obtained product $\mathbf{3 f}$ was crystallized in $n$-butanol and dried on $\mathrm{P}_{2} \mathrm{O}_{5}$ and was obtained in $54 \%$ yield as a colourless mass; m.p. 268-269 ${ }^{\circ} \mathrm{C}$. IR $\left(\mathrm{KBr}, v_{\max }, \mathrm{cm}^{-1}\right): 3300(\mathrm{O}-\mathrm{H}), 3220(\mathrm{~N}-\mathrm{H})$, 3030 (aromatic C-H), 2930 (aliphatic C-H), 1620-1560 (C=C and $\mathrm{C}=\mathrm{N}$ ), 800-640 (pyrimidine ring); ${ }^{1} \mathrm{H}$ NMR (400 MHz, DMSO- $\left.d_{6}\right): \delta=10.42(\mathrm{~s}, 1 \mathrm{H}$, pyrimidine $\mathrm{C}-\mathrm{H}), 7.76-6.95(\mathrm{~m}$, $8 \mathrm{H}, \mathrm{Ar}-\mathrm{H}), 5.84$ (s, 1H, O-H), 5.75, (s, 2H, N-H); 5.69 (s, 1H, $\mathrm{C}-\mathrm{H}), 3.89,3.87\left(\mathrm{~d}, 6 \mathrm{H}, 2 \times \mathrm{CH}_{3}\right) ;{ }^{13} \mathrm{C} \mathrm{NMR}(400 \mathrm{MHz}$, (DMSO- $\left.d_{6}\right): \delta=170.40(\mathrm{C}=\mathrm{S}$, pyrimidine ring), 147.15-111.40 (aromatic C), $75.07(\mathrm{CH}-\mathrm{OH}), 54.64\left(2 \times \underline{\mathrm{CH}}_{3} \mathrm{OC}_{6} \mathrm{H}_{4}^{-}\right)$. Anal. Calcd. for $\mathrm{C}_{19} \mathrm{H}_{19} \mathrm{~N}_{3} \mathrm{O}_{3} \mathrm{~S}$ : C, 61.77; H, 5.18; N, 11.37; S, 8.68. Found: C, 61.59; H, 5.02; N, 11.18; S, 8.80.

\section{RESULTS AND DISCUSSION}

Acetophenone semicarbazone and acetophenone thiosemicarbazone reacted with furan-2,3-diones and 1-methylenaminopyrimidine derivatives were synthesized. The $\mathrm{N}$-aminopyrimidine derivatives (1a-f) were used as important materials in the synthesis of the target heterocycles (Scheme-I) ${ }^{6-11}$. Briefly, the reactions of 1a-f were carried with 1,3-diketones and $\mathrm{NaBH}_{4}$.

In this study, the diazonium salts of $\mathbf{1 a - f}$ were condensed with dibenzoylmethane in the presence of $\mathrm{NaOH}$ with continuous stirring at $60-70{ }^{\circ} \mathrm{C}$ (Scheme-II). The reactions were monitored by thin-layer chromatography until complete consumption of the starting materials. After evaporation of the organic solvents and recrystallization from proper solvents (Experimental Section), 2a-f were obtained in moderate yields (50-67\%). The products 2 a-f obtained were characterized by FT IR, elementary analysis, ${ }^{1} \mathrm{H}$ NMR and ${ }^{13} \mathrm{C}$ NMR. In the IR spectrum of compound $\mathbf{2 a}$, the $\mathrm{C}=\mathrm{O}$ absorption peaks were seen at 1730,1680 and $1650 \mathrm{~cm}^{-1}$, respectively. In the ${ }^{1} \mathrm{H}$ NMR spectrum of compound $\mathbf{2 a}$, a peak observed at $\delta=14.90 \mathrm{ppm}$ was due to the enolic form. The ${ }^{1} \mathrm{H}$ NMR signals were found
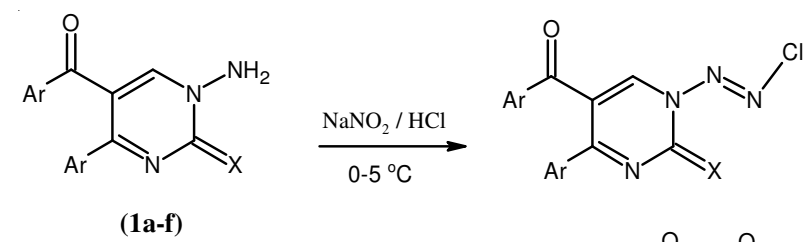

2a: $X=\mathrm{O}, \mathrm{Ar}=\mathrm{C}_{6} \mathrm{H}_{5}$
2b: $\mathrm{X}=\mathrm{S}, \mathrm{Ar}=\mathrm{C}_{6} \mathrm{H}_{5}$
2c: $\mathrm{X}=\mathrm{O}, \mathrm{Ar}=4-\mathrm{CH}_{3}-\mathrm{C}_{6} \mathrm{H}_{4}$
2d: $\mathrm{X}=\mathrm{S}, \mathrm{Ar}=4-\mathrm{CH}_{3}-\mathrm{C}_{6} \mathrm{H}_{4}$
2e: $\mathrm{X}=\mathrm{O}, \mathrm{Ar}=4-\mathrm{CH}_{3} \mathrm{O}-\mathrm{C}_{6} \mathrm{H}_{4}$
2f: $\mathrm{X}=\mathrm{S}, \mathrm{Ar}=4-\mathrm{CH}_{3} \mathrm{O}-\mathrm{C}_{6} \mathrm{H}_{4}$<smiles></smiles>

(2a-f)

$(50-67 \%)$

Scheme-II: Synthesis of compounds $\mathbf{2 a - f}$ 
at $\delta=7.36-7.02(\mathrm{~m}, 20 \mathrm{H}, \mathrm{ArH})$. The ${ }^{13} \mathrm{C}$ NMR signals were detected at 190.80-188.10 (benzoyl groups, $\mathrm{C}=\mathrm{O}$ ), 153.15 (pyrimidine ring, $\mathrm{C}=\mathrm{O}$ ), 150.05-110.42 (aromatic $\mathrm{C}$ ) and 74.82 $\mathrm{ppm}(\mathrm{CH})$. The elemental analysis data confirmed the structure of $\mathbf{2} \mathbf{a}$. The results of measurements of $\mathbf{2} \mathbf{b}-\mathbf{f}$ are given in the experimental section.

The reactions of $\mathrm{N}$-aminopyrimidine derivatives (1a-f) with sodium borohydride led to the formation of the corresponding 5-[hydroxy(phenyl)methyl]-4-phenylpyrimidine2(1H)-one derivatives (3a-f) in moderate yields (54-69\%) (Scheme-III). 1-Amino-5-[hydroxy(phenyl)methyl]-4-phenylpyrimidine-2(1H)-one (3a) was obtained in $54 \%$ yield by treating 1a with sodium borohydride and refluxing it in boiling ethanol for $1 \mathrm{~h}$. In the FT-IR spectrum of compound $\mathbf{3 a}$, the $\mathrm{OH}$ and $\mathrm{NH}_{2}$ absorption bands were found at 3300 and 3200 $\mathrm{cm}^{-1}$. The absorption peak of the carbonyl group was observed at about $1650 \mathrm{~cm}^{-1}$. The ${ }^{1} \mathrm{H}$ NMR signals were found at $\delta=$ $5.91(\mathrm{~s}, 1 \mathrm{H}, \mathrm{OH}), 5.80\left(\mathrm{~s}, 2 \mathrm{H}, \mathrm{NH}_{2}\right)$ and $5.74(\mathrm{~s}, 1 \mathrm{H}, \mathrm{CH})$. The ${ }^{1} \mathrm{H}$ NMR signals of the aromatic rings were found at $\delta=10.51$ (s, 1H, pyrimidine ring.) and 7.38-7.02 ppm (m, $10 \mathrm{H}, \mathrm{Ar}-\mathrm{H})$. The ${ }^{13} \mathrm{C}$ NMR signals were observed at $152.80(\mathrm{C}=\mathrm{O}$, pyrimidine ring), 143.15-117.42 (aromatic C) and 73.08 ppm $(\mathrm{CHOH})$. Finally, the elemental analysis data along with spectroscopic data confirmed the structure of compound $\mathbf{3 a}$. The other results of compounds $\mathbf{3 b - f}$ are given in the experimental section.

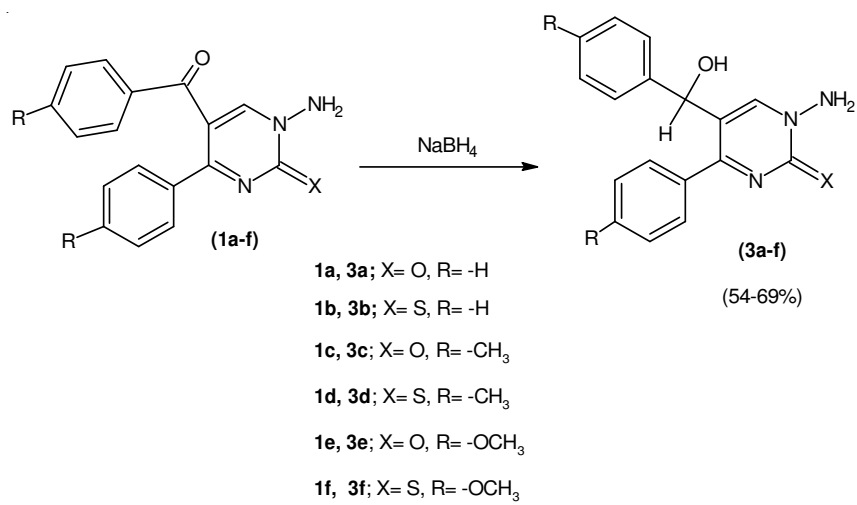

Scheme-III: A Synthetic pathway for the preparation of compounds $\mathbf{3 a - f}$

\section{ACKNOWLEDGEMENTS}

The autors are grateful for the financial support given by the Research Foundation of Erciyes University (Kayseri, Turkey).

\section{REFERENCES}

1. A.S. Galabov, E. Velichkova, A. Karparov, D. Sidzhakova, D. Danchev and N. Chakova, Arzneimittelforschung, 34, 9 (1984).

2. N. Azas, P. Rathelot, S. Djekou, F. Delmas, A. Gellis, C. Di Giorgio, P. Vanelle and P. Timon-David, Farmaco, 58, 1263 (2003).

3. P.F. Juby, T.W. Hudyma, M. Brown, J.M. Essery and R.A. Partyka, J. Med. Chem., 22, 263 (1979).

4. P.F. Juby, T.W. Hudyma, M. Brown, J.M. Essery and R.A. Partyka, J. Med. Chem., 25, 1145 (1982).

5. S. Raic-Malic, D. Svedruzic, T. Gazivoda, A. Marunovic, A. HergoldBrundic, A. Nagl, J. Balzarini, E. De Clercq and M. Mintas, J. Med. Chem., 43, 4806 (2000).

6. B. Altural, Y. Akçamur, E. Saripinar, I. Yildirim and G. Kollenz, Monatsh. Chem., 120, 1015 (1989).

7. Y. Akçamur, B. Altural, E. Saripinar, G. Kollenz, O. Kappe, E.M. Peters, E.-M. Peters and H.-G. Von Schnering, J. Heterocycl. Chem., 25, 1419 (1988).

8. E. Saripinar, Y. Guzel, Z. Onal, I.O. Ilhan and Y. Akcamur, J. Chem. Soc. Pak., 22, 308 (2000).

9. E. Saripinar, Ç. Yilmaz, D. Ünal, I. Özer Ilhan, N. Yazir and Y. Akçamur, Heterocycles, 68, 2045 (2006).

10. I. Yildirim and I. Koca, Kuwait J. Sci. Eng, 32, 49 (2005).

11. Z. Önal and I. Yildirim, Heterocycl. Commun., 13, 113 (2007).

12. Z. Önal, I. Yildirim, F. Kandemirli and T. Arslan, Struct. Chem., 21, 809 (2010).

13. Z. Önal, E. Saripinar and I.Ö. Ilhan, J. Heterocycl. Chem., 38, 397 (2001).

14. Z. Önal and B. Altural, Turk. J. Chem., 23, 401 (1999).

15. Z. Önal and A.C. Daylan, Asian J. Chem., 19, 2647 (2007).

16. Z. Önal, N. Atli and Ö.I. Ilhan, Heterocycl. Commun., 15, 385 (2009).

17. Z. Önal, H. Ceran and E. Sahin, Heterocycl. Commun., 14, 245 (2008). 